\title{
Inhibitors of zinc-dependent metalloproteases hinder sperm passage through the cumulus oophorus during porcine fertilization in vitro
}

\author{
J Beek, H Nauwynck'1' D Maes and A Van Soom \\ Departments of Reproduction, Obstetrics, and Herd Health and ${ }^{1}$ Virology, Parasitology and Immunology, Faculty of \\ Veterinary Medicine, Ghent University, B-9820 Merelbeke, Belgium
}

Correspondence should be addressed to J Beek; Email: josine.beek@ugent.be

\begin{abstract}
In this study, we report for the first time on a possible contribution of metalloproteases in sperm passage through the cumulus matrix in pigs. The presence of $20 \mu \mathrm{M}$ 1,10-phenanthroline (1,10-PHEN), inhibitor of zinc-dependent metalloproteases, strongly inhibited the degree of sperm penetration in cumulus-intact $(\mathrm{CI})$, but not in cumulus-free (CF), porcine oocytes during IVF. The inhibitory effect of 1,10-PHEN was due to the chelation of metal ions as a non-chelating analog (1,7-PHEN) did not affect IVF rates. Furthermore, incubation with 1,10-PHEN did not affect sperm binding to the zona pellucida nor sperm motility, membrane integrity, or acrosomal status.

These findings led to the assumption that 1,10-PHEN interacts with a sperm- or cumulus-derived metalloprotease. Metalloproteases are key players in physiological processes involving degradation or remodeling of extracellular matrix. In vivo, their proteolytic activity is regulated by tissue inhibitors of metalloproteases (TIMP1-TIMP4). We tested the effect of TIMP3 on fertilization parameters after porcine IVF. Similar to 1,10-PHEN, TIMP3 inhibited total fertilization rate of $\mathrm{CI}$ but not CF oocytes and did not influence sperm quality parameters. Although the inhibitory effect was stronger in $\mathrm{Cl}$ oocytes, TIMP3 also reduced the degree of sperm penetration in CF oocytes, suggesting the involvement of a metalloprotease in a subsequent step during fertilization. In conclusion, our results indicate the involvement of TIMP3-sensitive, zinc-dependent metalloprotease activity in sperm passage through the cumulus oophorus in pigs. The results should provide the basis for further biochemical research toward the localization and identification of the metalloprotease involved.

Reproduction (2012) 144 687-697
\end{abstract}

\section{Introduction}

During mammalian fertilization, the sperm cell interacts with the extracellular layers of the oocyte, the cumulus oophorus and the zona pellucida (ZP), to finally reach the oocyte plasma membrane. The cumulus oophorus consists of cumulus cells embedded in a matrix rich in hyaluronic acid. Traditionally, sperm hyaluronidases in combination with sperm motility are thought to enable spermatozoa to pass through the cumulus matrix and reach the ZP (Kim et al. 2008). The sperm binding to the $\mathrm{ZP}$ is then believed to induce the acrosome reaction with release of the acrosomal contzent, e.g. hydrolytic enzymes that serve in sperm penetration of the glycoprotein coat surrounding the oocyte, the ZP. However, due to recent findings, this accepted model of the acrosome reaction needs to be reevaluated. At least in the mouse, not only the ZP but also the cumulus oophorus appears to be a physiological site of the acrosome reaction (Yin et al. 2009, Jin et al. 2011, Sun et al. 2011).

Several proteases have been found on sperm membranes and in the acrosome of mammalian spermatozoa, including a collagenase-like peptidase, a cathepsin D-like protease, dipeptidyl peptidase II, (pro)acrosin, trypsin-like proteases other than acrosin, and testicular serine protease 5 (TESP5; Honda et al. 2002). In pigs, the sperm-specific serine protease acrosin (Polakoski \& McRorie 1973, Polakoski et al. 1973, Brown \& Cheng 1985) as well as the proteasome, a multi-subunit protease with specificity for ubiquitinated protein substrates, have been implicated in fertilization of cumulus-free (CF) oocytes (Sutovsky et al. 2004, Yi et al. 2007). Rather surprisingly, most of the studies on the role of proteases in mammalian fertilization did not evaluate sperm passage through the cumulus; although in most mammals, the cumulus oophorus is still present at the time of fertilization and can thus be considered as the first site of sperm-oocyte interaction (Van Soom et al. 2002). As a consequence, little is known about the functional significance of proteases in sperm passage through the cumulus oophorus. However, proteolytic activity may facilitate sperm passage through the viscous cumulus matrix by hydrolysis of bonds between hyaluronic acid and hyaluronic acid-binding proteins. 
Three families of metalloproteases are acknowledged to modify and degrade extracellular matrix, the matrix metalloproteases (MMPs), the 'A Disintegrin and Metalloproteinase' (ADAM) family of proteases, and the ADAM proteases with thrombospondin motifs (ADAMTS) (Shiomi et al. 2010). Their catalytic site typically contains a zinc binding motif and cleavage of substrates requires a zinc ion. The MMP family is mainly subdivided according to their domain structures and substrate specificity, e.g. collagenases (MMP1, MMP8, and MMP13), gelatinases (MMP2 and MMP9), and stromelysins (MMP3 and MMP10). Most of the MMPs are secreted, although there are at least six membrane-type MMPs that contain a transmembrane domain (Nagase et al. 2006). Some secreted MMPs can be associated with cells by interaction with cellular proteins. For example, MMP9 interacts with the hyaluronic acid receptor CD44 (Yu \& Stamenkovic 1999) and MMP2 can bind to integrin $\alpha v \beta 3$ (Brooks et al. 1996). The main function of MMPs has been considered to be the hydrolysis of matrix components. Next to that, MMPs may have a more complex role and function to disrupt cell-cell and cell-matrix interactions and mediate release of growth factors and cytokines (Stetler-Stevenson \& Yu 2001, Shiomi et al. 2010).

The diverse functions addressed to ADAMs can be explained by their typical structure containing a disintegrin domain and a metalloprotease domain (Reiss \& Saftig 2009). They are capable of mediating cell adhesion and migration via their disintegrin domain (e.g. integrin binding) as well as via proteolysis of cell adhesion molecules. ADAMs also have a prominent role in signaling pathways by cleavage of membrane-bound proteins and release of biologically active factors, such as growth factors. Most of the ADAMs are membraneanchored proteins but some members are secreted and are shown to cleave extracellular matrix components, such as ADAMTS1 and ADAMTS4 (Russell et al. 2003). Both ADAMTS1 and ADAMTS4 are produced and secreted by porcine cumulus cells (Shimada et al. 2004). It has been hypothesized that ADAMTS1mediated cleavage of versican, present in the cumulus matrix, may serve to stabilize the expanding matrix of the cumulus-oocyte complex (COC) by release of $70 \mathrm{kDa} N$-terminal fragment that binds to hyaluronic acid (Shimada et al. 2004). Studies on Adamts $1^{-1-}$ mice have demonstrated a lower cleavage of versican in expanded COCs, an impaired structural organization of the extracellular matrix, and also a delay in the degradation of the COC matrix after ovulation (Brown et al. 2010). Thus, ADAMTS1 may also function in the gradual shedding of cumulus cells and disassembly of the matrix after ovulation, which could enhance sperm penetration at the time of fertilization.

The objective of this study was to determine the possible involvement of metalloproteases during porcine fertilization in vitro. As metalloproteases are associated with breakdown and remodeling of extracellular matrix, we focused on IVF experiments with cumulusintact $(\mathrm{Cl})$ oocytes to evaluate the effect of metalloprotease inhibitors on sperm passage through the cumulus oophorus.

\section{Results \\ Fertilization parameters after porcine IVF are affected by 1,10-phenanthroline due to its capacity to chelate metal ions}

The possible involvement of metalloproteases in porcine fertilization was evaluated by assessing the inhibitory effect of two metalloprotease inhibitors, phosphoramidon and 1,10-phenanthroline (1,10-PHEN). Phosphoramidon is often used in the classification of newly identified metalloproteases (Beynon \& Bond 2001), although it has the limitation of targeting mainly bacterial metalloproteases and only few of mammalian origin (Bond \& Butler 1987). The metal ion chelator 1,10-PHEN can be used to inhibit zinc-dependent metalloproteases, without affecting the $\mathrm{Ca}^{2+}$ in the fertilization medium, as it has a much higher stability constant for $\mathrm{Zn}^{2+}$ than for $\mathrm{Ca}^{2+}$ (Correa et al. 2000). Both inhibitors were used at different concentrations within the range of effective concentrations reported by the manufacturer, and 1,10-PHEN was also used in lower concentrations.

The presence of phosphoramidon $(1-100 \mu \mathrm{M})$ during porcine fertilization in vitro did not affect total fertilization (control, $84.4 \%$ and phosphoramidon $100 \mu \mathrm{M}, 85.5 \%$ ) nor polyspermy rate (control, 45.4\% and phosphoramidon $100 \mu \mathrm{M}, 52.4 \%$ ). Furthermore, the sperm penetration index (SPI; mean number of

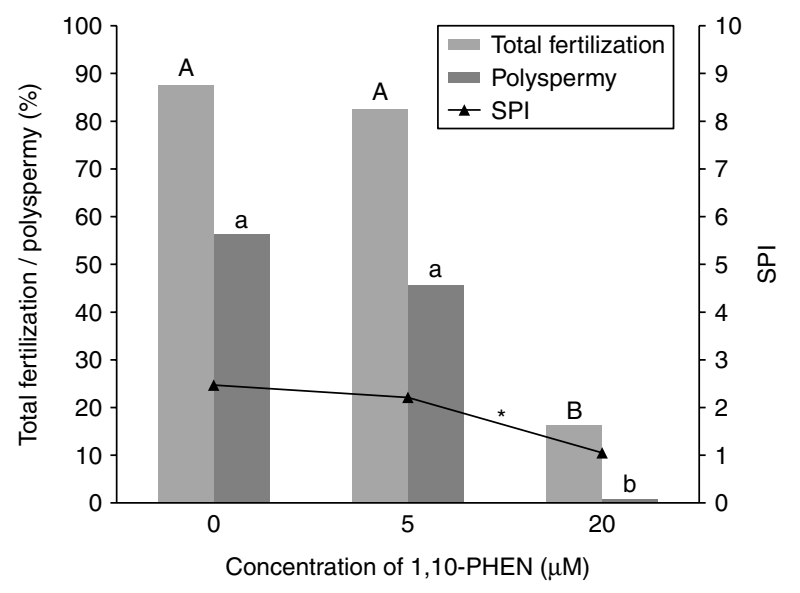

Figure 1 Effect of different concentrations of 1,10-PHEN ( 5 and $20 \mu \mathrm{M}$ ) on total fertilization, polyspermy rate, and the sperm penetration index (SPI) of cumulus-intact porcine oocytes. Data represent mean of three replicates. Values significantly different from control $(P<0.05)$ are marked with different capitals (total fertilization), different small letters (polyspermy), or an asterisk (SPI). 


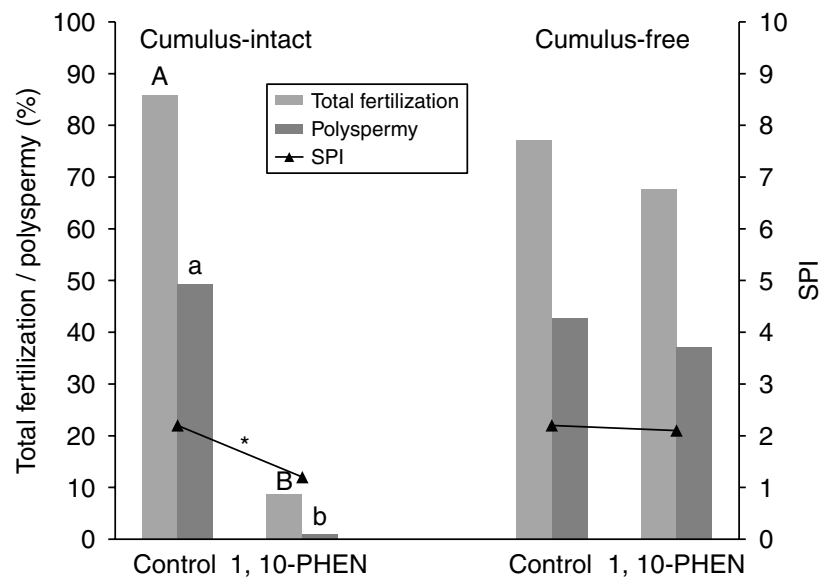

Figure 2 Effect of $20 \mu \mathrm{M}$ 1,10-PHEN on total fertilization, polyspermy rate, and the sperm penetration index (SPI) of cumulus-intact and cumulus-free porcine oocytes. Data represent mean of three replicates. Within each type of oocytes, values significantly different from control $(P<0.05)$ are marked with different capitals (total fertilization), different small letters (polyspermy), or an asterisk (SPI).

penetrated spermatozoa per fertilized oocyte) was very similar for COCs fertilized in control medium and medium with $100 \mu \mathrm{M}$ phosphoramidon, 2.29 and 2.31 respectively. In contrast, 1,10-PHEN $(20 \mu \mathrm{M})$ was found to strongly decrease both total and polyspermic fertilization rate compared with the control group $(P<0.05 ;$ Fig. 1$)$. The lowest concentration of 1,10-PHEN with a significant effect on fertilization rate was $20 \mu \mathrm{M}$. Therefore, this concentration was used in the subsequent experiments with 1,10-PHEN.

To evaluate the effect of 1,10-PHEN on sperm penetration of the cumulus oophorus, we compared fertilization parameters of $\mathrm{Cl}$ and $\mathrm{CF}$ oocytes. The results showed a striking difference in inhibition of total fertilization rate between $\mathrm{Cl}$ and $\mathrm{CF}$ oocytes (Fig. 2). Total fertilization rate of $\mathrm{Cl}$ oocytes was inhibited with $90 \%$ compared with the respective control group, whereas a very small and no significant inhibition was recorded after denudation of oocytes. In $\mathrm{Cl}$ oocytes, polyspermic fertilization was markedly reduced by
1,10-PHEN $126(P<0.01)$, whereas in CF oocytes this parameter was not different from the control group ( $P=0.469$ and $P=0.427$ respectively). Furthermore, the SPI was significantly reduced by 1,10 -PHEN in $\mathrm{Cl}$ oocytes, 1.2 (1,10-PHEN) vs 2.2 (control), but not in CF oocytes (SPI 2.2 and 2.1). When COCs were co-incubated with mitotracker-labeled spermatozoa in fertilization medium with and without $20 \mu \mathrm{M}$ $1,10-\mathrm{PHEN}$, less spermatozoa were observed in the inner layers of the cumulus oophorus of COCs fertilized in medium with 1,10-PHEN (Fig. 3).

1,10-PHEN did not substantially affect sperm binding to the ZP of CF oocytes. After $4 \mathrm{~h}$ of gamete co-incubation, the average number of spermatozoa bound to the ZP ( \pm s.D.) was $92 \pm 26$ in the control group and $73 \pm 21$ in the group of $1,10-\mathrm{PHEN}$. The high number of spermatozoa that bound to the ZP in this experiment is dependent on the sperm concentration in the fertilization droplet (Fazeli et al. 1995). The major fraction of these spermatozoa would not succeed in penetration of the ZP. Together, these findings indicate that the inhibitory effect of 1,10-PHEN is predominantly situated at the level of sperm interaction with the cumulus.

In order to confirm that the inhibitory effect of 1,10-PHEN was mediated by its capacity to bind metal ions, $\mathrm{Cl}$ and $\mathrm{CF}$ oocytes were fertilized in medium with and without $20 \mu \mathrm{M}$ of a non-chelating analog, $1,7-\mathrm{PHEN}$. There were no significant differences in fertilization parameters between either $\mathrm{Cl}$ or CF oocytes fertilized in standard medium or medium with $20 \mu \mathrm{M}$ 1,7-PHEN (Fig. 4). As 1,10-PHEN mainly targets zinc, we hypothesized the involvement of a zinc-dependent metalloprotease in sperm passage through the cumulus oophorus during porcine IVF.

\section{1,10-PHEN has no significant effect on membrane integrity, motility, and acrosomal status of porcine spermatozoa}

To exclude a possible negative influence of 1,10-PHEN on the spermatozoa, parameters related to the fertilizing
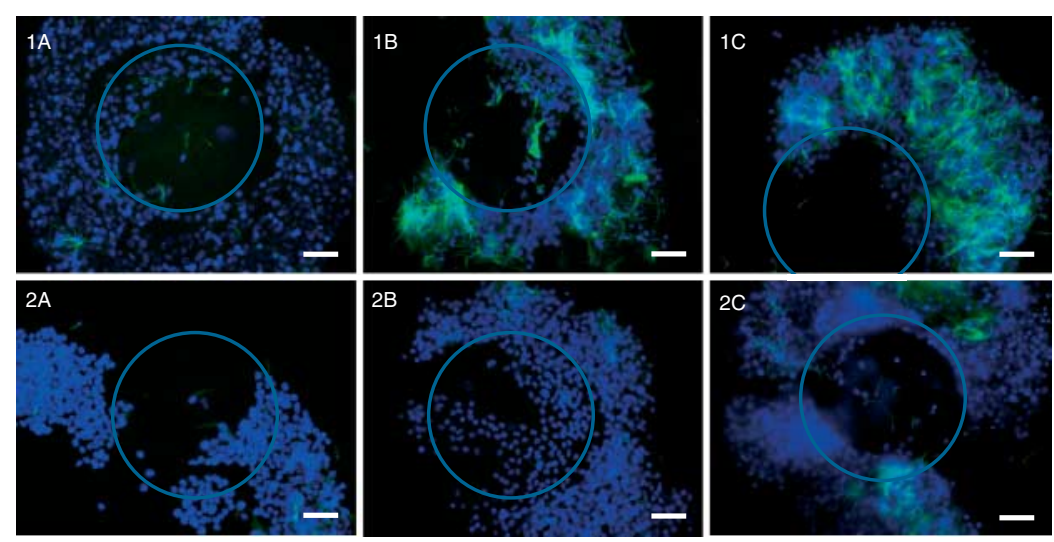

Figure 3 Visualization of spermatozoa within the cumulus oophorus after $2 \mathrm{~h}(\mathrm{~A}), 4 \mathrm{~h}(\mathrm{~B})$, and $6 \mathrm{~h}(\mathrm{C})$ of gamete co-incubation in (1) standard medium and (2) medium with $20 \mu \mathrm{M}$ 1,10-PHEN. The outline of the oocytes is represented by a blue circle. Spermatozoa were labeled by Mitotracker Green, nuclei were stained with Hoechst (original magnification, 400×; Bar $=25 \mu \mathrm{m})$. 


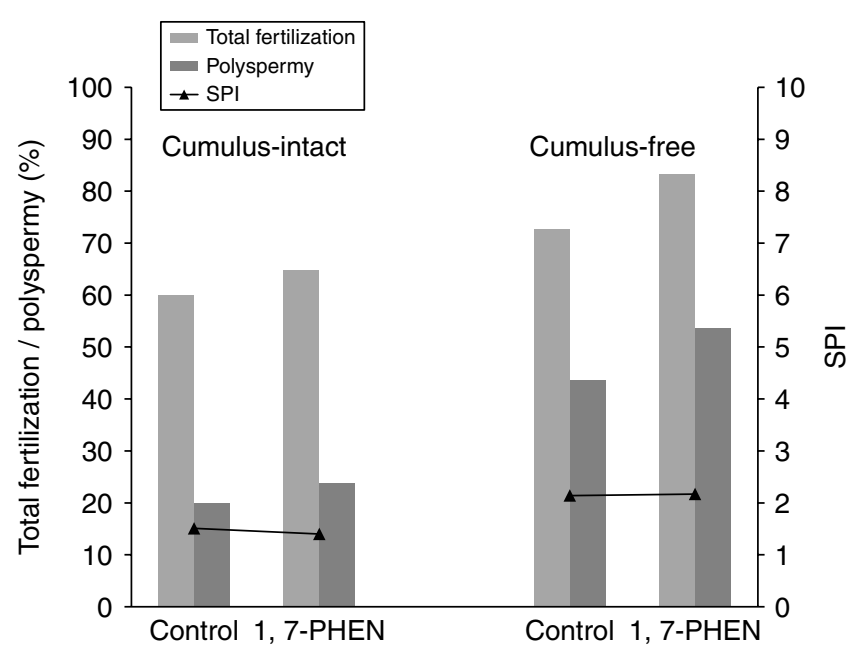

Figure 4 Effect of $20 \mu \mathrm{M}$ 1,7-PHEN on total fertilization, polyspermy rate, and the sperm penetration index (SPI) of cumulus-intact and cumulus-free porcine oocytes. Data represent mean of three replicates. Within each type of oocytes, none of the parameters were significantly different between control and treatment group $(P>0.05)$.

capacity of spermatozoa were evaluated during a 6 -h incubation period in medium supplemented with and without $20 \mu \mathrm{M} 1,10-\mathrm{PHEN}$. Sperm membrane integrity and motility were not affected by 1,10-PHEN as shown in Fig. 5A and $\mathrm{B}$ respectively. Next to that, the acrosomal status of frozen-thawed epididymal spermatozoa incubated in $20 \mu \mathrm{M} 1,10$-PHEN was not significantly different $(P>0.05)$ from that of spermatozoa in control medium (Fig. 5C). After $1 \mathrm{~h}$ of incubation with $1 \mu \mathrm{M}$ A23187 calcium ionophore, the percentage of spermatozoa that underwent the acrosome reaction was similar in both groups. In the control group, $9.3 \pm 3.1 \%$ of the spermatozoa remained acrosome intact vs $9.5 \pm 3.1 \%$ of acrosome-intact spermatozoa in medium with $20 \mu \mathrm{M}$ 1,10-PHEN.

\section{Evidence for the involvement of a tissue inhibitor of metalloprotease 3-sensitive, zinc-dependent metallo- protease in sperm passage through the cumulus oophorus during porcine IVF}

Here, we aimed to assess whether the effect of 1,10 -PHEN on sperm passage through the cumulus oophorus could be due to inhibition of a zinc-dependent metalloprotease involved in matrix breakdown and remodeling. In vivo, tissue inhibitors of metalloproteases (TIMPs) strictly control all active forms of MMPs. Four related TIMPs (TIMP1-TIMP4) have each the capacity to form a 1:1 complex with all MMPs, but there is variation in the strength of inhibition. Additionally, it has been shown that TIMPs can also inhibit some members of the ADAM and ADAMTS family of metalloproteases. We tested recombinant human TIMP3 $(400 \mathrm{nM})$ and evaluated the fertilization parameters after IVF of $\mathrm{Cl}$ and CF oocytes (Fig. 6). The presence of $400 \mathrm{nM}$ TIMP3 during gamete co-incubation decreased total fertilization rate of $\mathrm{Cl}$ oocytes $(P<0.01)$. In contrast, when $\mathrm{CF}$ oocytes were used, there was no significant difference in total fertilization rate between the control group and TIMP3 $(P=0.166)$. Polyspermic fertilization was markedly reduced in both $\mathrm{Cl}$ and $\mathrm{CF}$ oocytes by incubation with TIMP3 during IVF $(P<0.01)$. There was also a
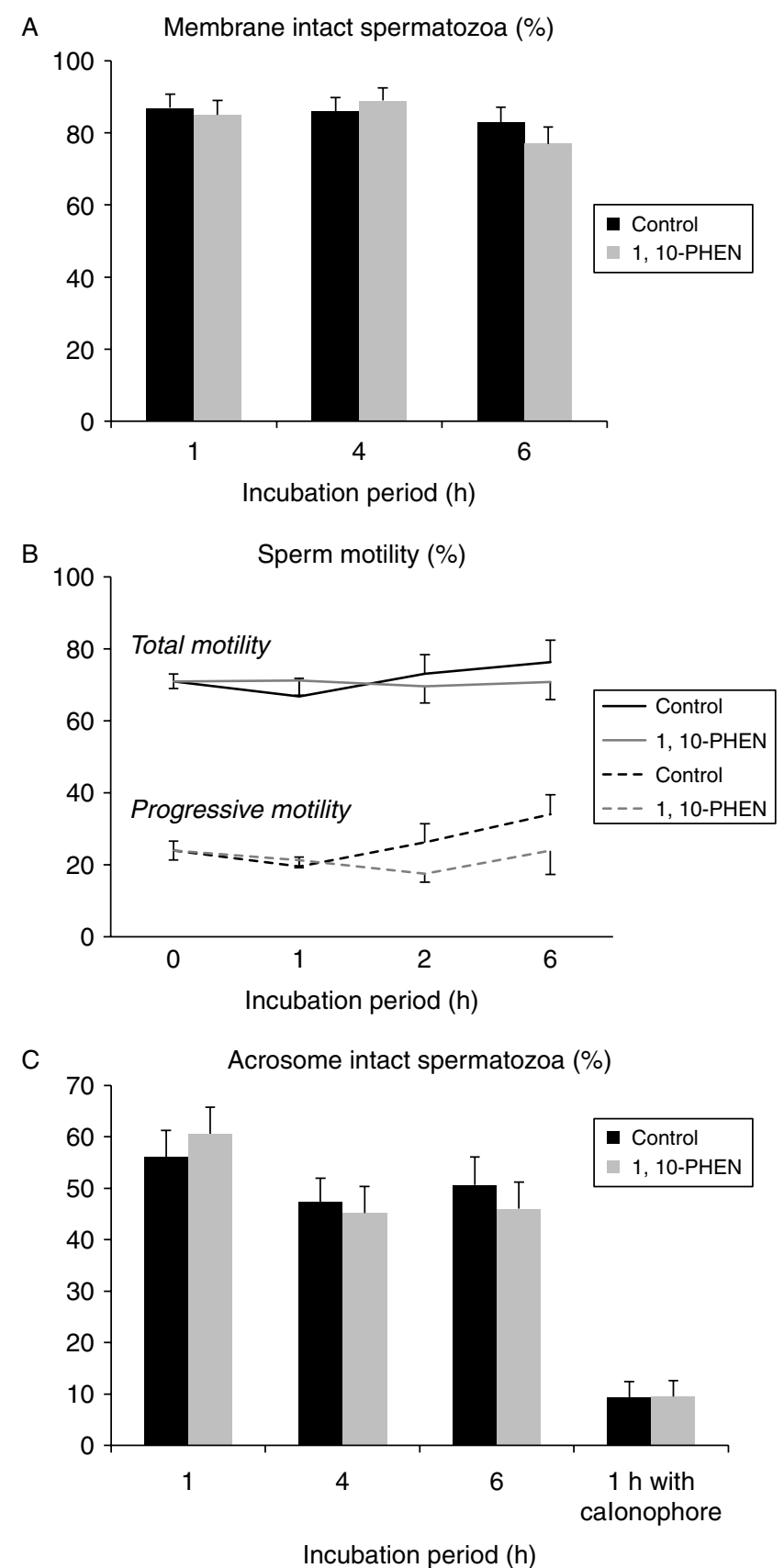

Figure 5 Effect of $20 \mu \mathrm{M}$ 1,10-PHEN on membrane integrity (A), motility (B), and acrosomal integrity (C) of porcine spermatozoa at different time points within a 6 -h incubation period. Data represent mean \pm S.E.M. of three replicates. None of the parameters were significantly different between control and treatment group at any of the evaluated time points $(P>0.05)$. 


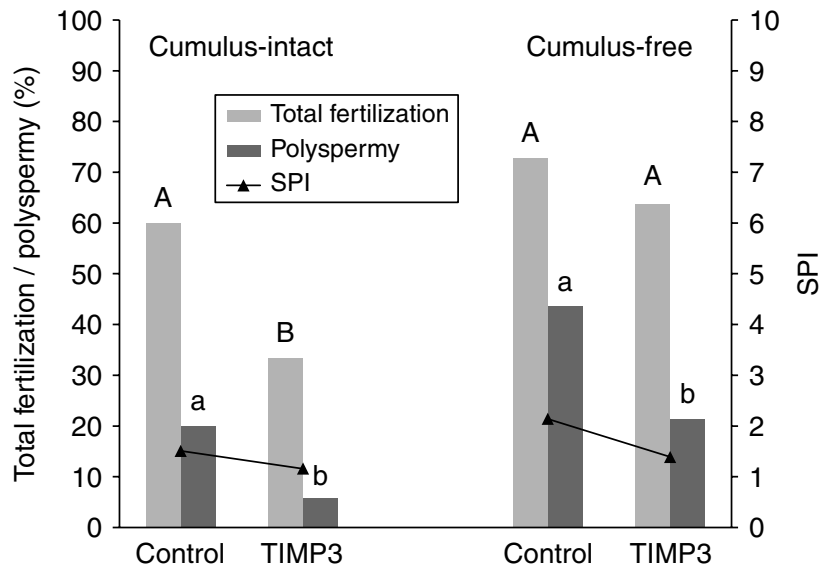

Figure 6 Effect of $400 \mathrm{nM}$ TIMP3 on total fertilization, polyspermy rate, and the sperm penetration index (SPI) of cumulus-intact and cumulusfree porcine oocytes. Data represent mean of three replicates. Within each type of oocytes, values significantly different from control $(P<0.05)$ are marked with different capitals (total fertilization) or different small letters (polyspermy). There were no significant differences in the sperm penetration index.

tendency toward a lower SPI in the presence of TIMP3, yet the differences were not statistically significant (Cl oocytes, 1.5 (control) vs 1.2 (TIMP3); CF oocytes, 2.1 (control) vs 1.4 (TIMP3)). In conclusion, TIMP3 reduces the degree of sperm penetration in both $\mathrm{Cl}$ and CF oocytes, with a negative effect on total fertilization rate in $\mathrm{Cl}$ oocytes but not in CF oocytes.

\section{TIMP3 has no significant effect on membrane integrity, motility, and acrosomal status of porcine spermatozoa}

To exclude a possible negative influence of TIMP3 on the spermatozoa, membrane integrity, motility, and the acrosomal status of spermatozoa were evaluated at different time points during $6 \mathrm{~h}$ of incubation. Sperm incubation with $400 \mathrm{nM}$ TIMP3 did not significantly affect membrane integrity or sperm motility at any of the evaluated time points ( $P>0.05$; Fig. $7 \mathrm{~A}$ and $\mathrm{B})$. The acrosomal status of frozen-thawed epididymal spermatozoa in medium supplemented with $400 \mathrm{nM}$ TIMP3 was not significantly different from that of spermatozoa incubated in standard fertilization medium $(P>0.05$; Fig. 7C). After $1 \mathrm{~h}$ of incubation with $1 \mu \mathrm{M}$ A23187 calcium ionophore, the fraction of spermatozoa that underwent the acrosome reaction was similar in both groups.

\section{Discussion}

The functional significance of proteases for sperm passage through the cumulus matrix is largely unknown. Sperm hyaluronidases on the contrary, in combination with sperm motility, have been considered imperative in this step of the fertilization process (Primakoff \& Myles 2002, Kim et al. 2008). In this study, we report for the first time on a possible contribution of metalloproteases in sperm passage through the cumulus matrix in pigs. We based our conclusions on several observations: 1) inhibition of zinc-dependent metalloproteases, by means of 1,10-PHEN, induced a strong inhibitory effect on the degree of sperm penetration in $\mathrm{Cl}$, but not in $\mathrm{CF}$ porcine oocytes; 2) the inhibitory effect of 1,10-PHEN
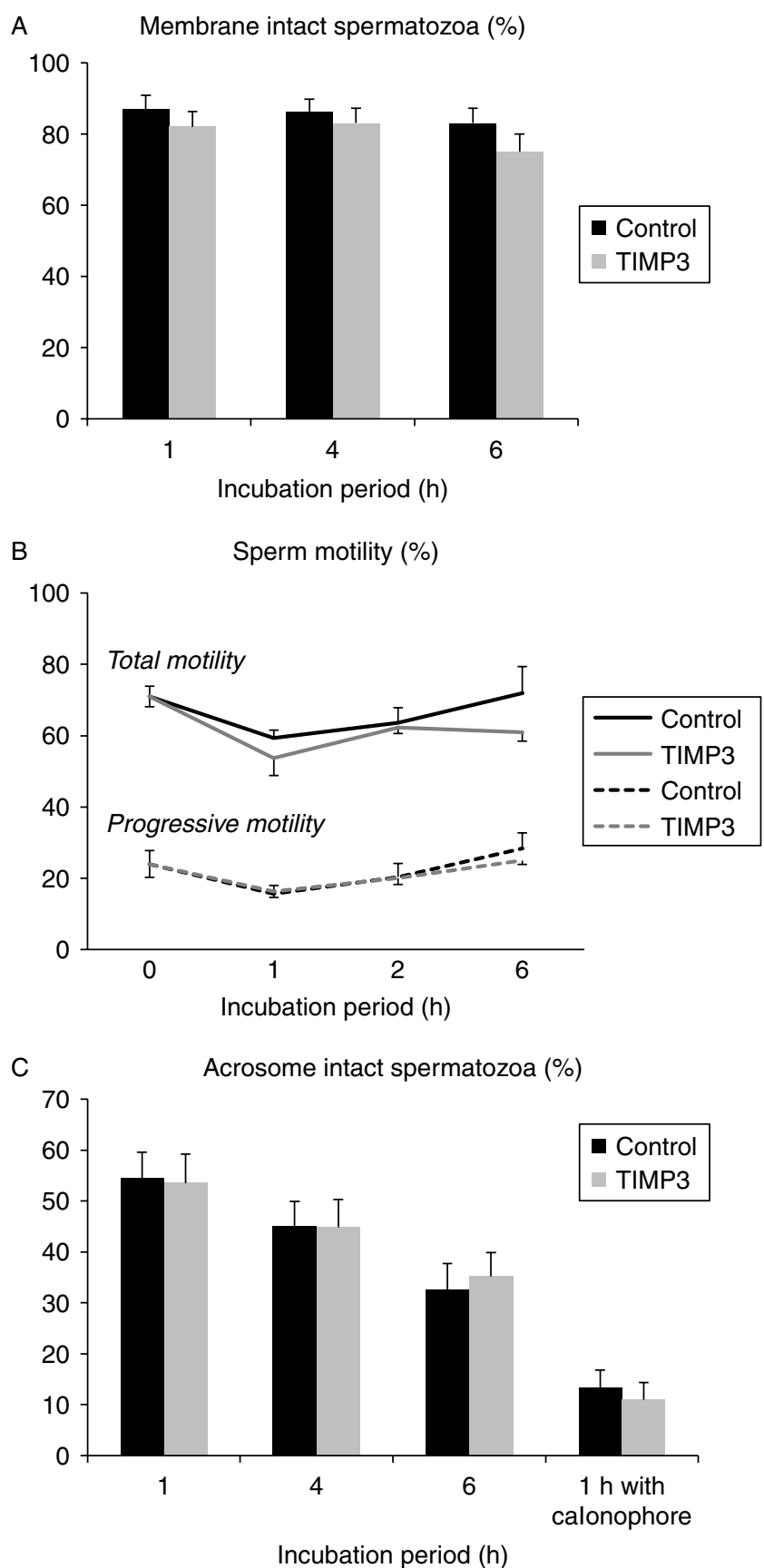

Figure 7 Effect of $400 \mathrm{nM}$ TIMP3 on membrane integrity (A), motility (B), and acrosomal integrity (C) of porcine spermatozoa at different time points within a 6-h incubation period. Data represent mean \pm s.E.M. of three replicates. None of the parameters were significantly different between control and treatment group at any of the evaluated time points $(P>0.05)$. 
was due to the chelation of metal ions as a non-chelating analog (1,7-PHEN) did not affect IVF rates; 3) MMP inhibitor TIMP3 showed a stronger inhibitory effect on total fertilization and sperm penetration rate in $\mathrm{Cl}$ oocytes than in CF oocytes, similar to 1,10-PHEN; and 4) the effect of the inhibitors was not mediated by a decrease in sperm quality as sperm motility, membrane integrity, and acrosomal status were not altered.

Whereas the inhibition profile of 1,10-PHEN is not specific (inhibition of all zinc-dependent metalloproteases via chelation of the catalytic zinc atom), the inhibitory effect by TIMP3 points to the family of MMPs and some members of the ADAM family of metalloproteases that are inhibited by TIMP3.

Only few studies have looked into sperm-derived metalloprotease activity thus far. Buchman-Shaked et al. (2002) described the localization of MMP2 at the sperm head of human spermatozoa and release of MMP2 from spermatozoa during a short period after incubation in capacitation medium. The presence of MMP2 on spermatozoa of other species, including the pig, remains putative. Expression of ADAM1 and ADAM2 in porcine spermatozoa, on the other hand, has recently been documented (Fabrega et al. 2011). These two ADAMs form a hetero-dimeric complex 'fertilin' that was initially isolated at the sperm surface of guinea pig spermatozoa (Primakoff et al. 1987) and participates in sperm-oocyte interaction via binding to integrins (Evans 2001). Yet ADAM1 and ADAM2 are not considered as candidate enzymes for the observed effect of 1,10-PHEN as both members of the ADAM family are enzymatically inactive and therefore unsusceptible for zinc deprivation. From the eight ADAM genes identified in the pig, ADAM8, ADAM9, ADAM10, ADAM15, and ADAM17 encode for a protein with metalloprotease activity (Rawlings et al. 2012). TIMP3 has been shown to inhibit ADAM10 and ADAM17 (Amour et al. 1998, 2000, Loechel et al. 2000) but not ADAM8 and ADAM9 (Amour et al. 2002). It is not yet known whether TIMP3 is able to inhibit ADAM15. Especially, ADAM10 and ADAM17 are of interest as putative sperm-derived metalloproteases because they mediate cleavage of cell adhesion molecules such as L-selectin, cadherins, and also CD44 (Peschon et al. 1998, Nagano \& Saya 2004, Nagano et al. 2004, Maretzky et al. 2005). CD44 is present on the surface of cumulus cells of in vivo- and in vitro-matured porcine COCs and attaches the cumulus cells to the matrix by interaction with hyaluronic acid (Yokoo et al. 2002, 2007). A downstream effect of CD44 cleavage is cell detachment from extracellular matrix (Nagano \& Saya 2004). One of the hypotheses we derived from this study is that the TIMP3-sensitive metalloprotease activity involved in sperm passage through the cumulus oophorus might be directed toward CD44 on cumulus cells (in order to enhance cell detachment and facilitate sperm passage). In that respect, we speculate that a TIMP3-sensitive ADAM, e.g. ADAM10 or ADAM17, may be present on spermatozoa. These are for now only speculations and will have to be tested in further research. The use of immunofluorescent staining techniques could be a first approach to investigate whether specific ADAMs are present on spermatozoa or not.

Proteases are also abundantly secreted by cumulus cells. For example, urokinase plasminogen activator, tissue plasminogen activator, ADAM17, ADAMTS1, and ADAMTS4 have been shown to be synthesized and secreted by cumulus cells (D'Alessandris et al. 2001, Shimada et al. 2004, Yamashita et al. 2007). The proteolytic activity of ADAMTS1 has been associated with expansion of the cumulus matrix and remodeling of the follicle wall before ovulation (Russell et al. 2003, Shimada et al. 2004, Brown et al. 2010), but has also a critical function in the timely degradation of the cumulus matrix after ovulation. COCs of Adamts $1^{-1-}$ mice were shown to retain cumulus cells, versican, and hyaluronic acid after ovulation (Brown et al. 2010). In addition, Adamts $1^{-1-}$ females showed a lower IVF rate. Brown et al. proposed that reduced clearance of versican could lead to a more resistant cumulus matrix and inhibition of sperm penetration. In our study, it became clear that the addition of the metalloprotease inhibitor during porcine IVF reduced sperm passage through the cumulus. It is described that porcine cumulus cells increase production of ADAMTS1 in response to gonadotropins, followed by incorporation of ADAMTS1 in the cumulus matrix (Shimada et al. 2004). It has been shown that TIMP3 is able to inhibit ADAMTS1, ADAMTS2, ADAMTS4, and ADAMTS5 (Kashiwagi et al. 2001, Rodriguez-Manzaneque et al. 2002, Wang et al. 2006). Based on these data from the literature, it seems theoretically probable that, in our study, inhibition of cumulus-derived metalloproteases (especially inhibition of ADAMTS1) could have hampered physiological degradation of the cumulus matrix. Inferior matrix disassembly may become evident by the tendency of cumulus cells to stick to the bottom of the culture dish: Shimada et al. (2004) noticed a similar phenomenon after culture of porcine COCs in medium with the MMP inhibitor Galardin. We did observe release of cumulus cells from the outer layers of the cumulus oophorus followed by adherence of cumulus cells to the culture dish when COCs were fertilized in medium with the inhibitor 1,10-PHEN. However, the number of cumulus cell layers that surrounded the oocytes after $6 \mathrm{~h}$ of gamete co-incubation was not affected by the presence or absence of the inhibitor 1,10-PHEN, whereas the number of spermatozoa that had reached the inner layers of the cumulus oophorus at that time was noticeably lower in the presence of 1,10-PHEN, as shown via fluorescent labeling (Fig. 3). In another experiment, we found that preincubation of COCs with 1,10-PHEN during 30 min before IVF had no significant effect on fertilization parameters (Table 1), although the level of 
cumulus-derived ADAMTS1 protein in porcine COCs reaches its maximum at the end of the maturation period (Shimada et al. 2004). As a result, we had to reject the hypothesis that in our study metalloprotease inhibitors strongly decreased the degree of sperm penetration due to inhibition of cumulus-derived metalloproteases and hindrance of physiological matrix degradation.

Based on our findings, the effect of 1,10-PHEN was clearly restricted to fertilization of $\mathrm{Cl}$ oocytes. However, TIMP3 showed a more complex inhibitory effect. TIMP3 inhibited total fertilization rate in $\mathrm{Cl}$ and not in $\mathrm{CF}$ oocytes, similar to 1,10-PHEN. Yet TIMP3 reduced the degree of sperm penetration in $\mathrm{Cl}$ as well as CF oocytes. These results indicate that the general effect of TIMP3 is a reduction in the number of sperm entering an oocyte. Because the effect is stronger in $\mathrm{Cl}$ than in CF oocytes, this still points to an inhibitory effect at the level of sperm-cumulus interaction. In addition, TIMP3 seems to inhibit a subsequent step in the fertilization process. Correa et al. (2000) showed that in mice, a TIMP3sensitive metalloprotease is involved in sperm-oocyte fusion. Based on the effect of a number of metalloprotease inhibitors, they hypothesized a role for a MMP or ADAM metalloprotease of which the identity still needs to be unraveled (Correa et al. 2000). In the pig, ADAM1 and ADAM2 are present on the sperm membrane (Fabrega et al. 2011). To answer whether the reduction in number of sperm entering CF porcine oocytes by TIMP3 could be due to inhibition of ADAM1 or ADAM2, the inhibition profile of TIMP3 needs further investigation.

In conclusion, this study opens new possibilities to improve our understanding of the fertilization process and should provide a basis for further research toward the localization and identification of the metalloprotease involved in sperm passage through the cumulus oophorus. The recognition of key proteases in the fertilization process holds interesting opportunities for porcine embryo production in vitro (IVP). Specific inhibitors may be useful as regulators of sperm penetration in porcine IVF, lessening the problem of polyspermic fertilization and increasing IVP efficiency.

Table 1 Fertilization parameters of cumulus-intact porcine oocytes inseminated with $1.25 \times 10^{5}$ spermatozoa $/ \mathrm{ml}$ in standard fertilization medium after 30 min of oocyte preincubation in fertilization medium with and without supplementation of $20 \mu \mathrm{M}$ 1,10-PHEN.

\begin{tabular}{lcccc}
\hline Group & $\begin{array}{c}\text { Total } \\
\text { fertilization } \\
(\%)\end{array}$ & $\begin{array}{c}\text { Mono- } \\
\text { spermy } \\
(\%)\end{array}$ & $\begin{array}{c}\text { Polyspermy } \\
(\%)\end{array}$ & $\begin{array}{c}\text { Sperm } \\
\text { penetration } \\
\text { index }^{\mathrm{a}}\end{array}$ \\
\hline Control & 76.4 & 55.0 & 21.4 & 1.3 \\
$1,10-$ PHEN & 73.2 & 54.2 & 19.0 & 1.5 \\
\hline
\end{tabular}

Number of oocytes examined: control, $n=104 ; 1,10-\mathrm{PHEN}, n=96$ Data represent mean of two replicates. None of the parameters were significantly different between control and treatment group $(P>0.05)$. ${ }^{a}$ Mean number of penetrated spermatozoa per fertilized oocyte.

\section{Materials and Methods}

\section{Media}

All chemicals used in this study were purchased from Sigma-Aldrich, unless otherwise stated. The basic medium used for the collection and washing of COCs was a modified HEPES-buffered Tyrode balanced salt solution (HEPES-TM) with $10 \mu \mathrm{g} / \mathrm{ml}$ gentamicin sulfate, $10 \mathrm{mM}$ HEPES, and $3 \mathrm{mg} / \mathrm{ml}$ BSA. Immature oocytes were matured in BSA-free 'North Carolina State University 23' (NCSU23; Petters \& Wells 1993) supplemented with $0.57 \mathrm{mM}$ cysteine, $10 \mathrm{ng} / \mathrm{ml}$ epidermal growth factor, $10 \mathrm{IU} / \mathrm{ml}$ equine chorionic gonadotropin (Folligon, Intervet, Boxmeer, The Netherlands), $10 \mathrm{IU} / \mathrm{ml}$ human chorionic gonadotropin (Chorulon, Intervet), and $10 \%$ porcine follicular fluid. Follicular fluid was collected from 5 to $6 \mathrm{~mm}$ follicles of prepubertal gilts. After centrifugation of follicular fluid $(100 \mathrm{~g}, 10 \mathrm{~min})$, the supernatant was aspirated, filtered $(0.22 \mu \mathrm{m})$, and stored at $-80{ }^{\circ} \mathrm{C}$ until use. The basic medium for IVF was Tyrode's albumin lactate pyruvate (TALP) medium (Rath et al. 1999) supplemented with $0.3 \%$ BSA (FERT-TALP). Frozen-thawed epididymal boar spermatozoa were centrifuged ( $390 \mathrm{~g}, 3 \mathrm{~min}$ ) in Androhep extender consisting of $27 \mathrm{mM}$ Tri-natriumcitraat, $2.7 \mathrm{mM}$ Titriplex III EDTA, $2.5 \mathrm{mg} / \mathrm{ml}$ BSA (fraction V),

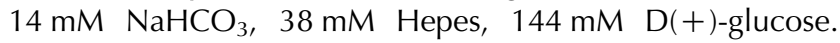
$\mathrm{H}_{2} \mathrm{O}$, and $50 \mu \mathrm{g} / \mathrm{ml}$ gentamicin sulfate. Presumed zygotes were washed in HEPES-buffered TALP (HEPES-TALP) medium, i.e. TALP medium with $25 \mathrm{mM}$ HEPES. The embryo culture medium was NCSU23 with $0.4 \%$ BSA.

\section{Protease inhibitors}

Protease inhibitors were purchased from Sigma-Aldrich and used at different concentrations. Both 1,10-PHEN (P9375) and 1,7-PHEN (301841) were dissolved in methanol (stock solution, $200 \mathrm{mM}$ ). Phosphoramidon was dissolved in FERT-TALP medium $(500 \mu \mathrm{M})$ and recombinant human TIMP3 was dissolved in deionized water (1000 nM).

\section{Oocyte collection}

Ovaries of prepubertal gilts were collected at a local slaughterhouse and prepared following the protocol of Bijttebier et al. (2008). Briefly, immature COCs were aspirated from follicles with a diameter ranging from 3 to $6 \mathrm{~mm}$. Only COCs with a homogeneous ooplasm and a multilayered cumulus were selected.

\section{In vitro maturation and IVF of porcine COCs}

Groups of 100 immature COCs were placed into $500 \mu \mathrm{l}$ maturation medium and cultured for the first $22 \mathrm{~h}\left(39^{\circ} \mathrm{C}, 5 \%\right.$ $\mathrm{CO}_{2}$ ). Subsequently, COCs were cultured in hormone-free maturation medium for $22 \mathrm{~h}$. After in vitro maturation of COCs, protease inhibitor dilutions were prepared in concentrated stocks and added to droplets of FERT-TALP to obtain the final concentration required. As TIMP3 was dissolved in deionized water, the concentration of components in the medium was 
adjusted by adding FERT-TALP-2 $X$ in the same volume as the stock solution of TIMP3 to each fertilization droplet. Subsequently, the COCs were randomly assigned to the fertilization droplets (50 COCs/droplet). Frozen epididymal semen was thawed for $60 \mathrm{~s}$ in a water bath at $38{ }^{\circ} \mathrm{C}$ and spermatozoa were washed by centrifugation ( $3 \mathrm{~min}$ at $390 \mathrm{~g}$ ) in $9.5 \mathrm{ml}$ Androhep. The sperm pellet was resuspended in $1 \mathrm{ml}$ FERT-TALP and sperm concentration was assessed using a Bürker counting chamber. Spermatozoa were added to the droplets containing the COCs resulting in a final concentration of $1.25 \times 10^{5}$ spermatozoa $/ \mathrm{ml}$. The control group consisted of COCs fertilized under standard conditions. After a co-incubation period of $6 \mathrm{~h}$, the presumed zygotes were vortexed during $3 \mathrm{~min}$ in $2.5 \mathrm{ml}$ HEPES-TALP to remove loosely bound spermatozoa, washed three times in culture medium, and cultured for $18 \mathrm{~h}$ in a modular incubator chamber $\left(39^{\circ} \mathrm{C}, 5 \% \mathrm{CO}_{2}\right)$.

\section{Assessment of fertilization parameters}

Approximately $24 \mathrm{~h}$ after insemination, presumed zygotes were washed in $0.1 \%(\mathrm{w} / \mathrm{v})$ polyvinylpyrrolidone in PBS. Subsequently, the zygotes were fixed overnight with $4 \%$ paraformaldehyde in PBS and stained with $10 \mu \mathrm{g} / \mathrm{ml}$ bis-benzamide (Hoechst 33342; Molecular Probes, Leiden, The Netherlands) for $10 \mathrm{~min}$. Zygotes were mounted in a droplet of glycerol with $(25 \mathrm{mg} / \mathrm{ml})$ 1,4-diazabicyclo (2.2.2) octane (DABCO; Acros, Ghent, Belgium) and nuclear DNA was visualized using a Leica DMR fluorescence microscope (Leica Microsystems, Brussels, Belgium). Oocytes with a metaphase plate and a polar body were classified as being in the MII stage. The presence of two pronuclei or cleaved embryos with two equally sized blastomeres was indicative for fertilization, whereas oocytes without penetrated sperm heads were considered as not fertilized. Zygotes with more than two pronuclei or more than one decondensed sperm head were classified as polyspermic. In case of polyspermic fertilization, the number of penetrated spermatozoa was counted. The following fertilization parameters were assessed: total fertilization rate (\%), polyspermy (\%), and the SPI (mean number of penetrated spermatozoa per fertilized oocyte).

\section{Effect of metalloprotease inhibitors on fertilization parameters after porcine IVF}

COCs were fertilized in the presence of 1,10-PHEN (5 and $20 \mu \mathrm{M} ; n=483$ ) or phosphoramidon (10 and $100 \mu \mathrm{M} ; n=453$ ). Control COCs were fertilized under standard conditions ( $n=251$ and $n=245$ for the experiments with 1,10-PHEN and phosphoramidon respectively). Each experimental unit consisted of two droplets of 40-50 oocytes. This experiment was repeated three times for each protease inhibitor.

\section{Effect of metalloprotease inhibitors on fertilization parameters after IVF of $\mathrm{Cl}$ vs CF porcine oocytes}

In vitro matured COCs were randomly assigned to eight groups of $\sim 40$ oocytes each (three replicates). Half of the COCs (four groups) were denuded by vortexing with $0.1 \%(\mathrm{w} / \mathrm{v})$ hyaluronidase in HEPES-TM (cumulus free or CF), the other half were kept $\mathrm{Cl}$. Both $\mathrm{CF}$ and $\mathrm{Cl}$ oocytes were fertilized under standard conditions $(n=291)$ and in the presence of 1,10-PHEN (20 $\mu \mathrm{M} ; n=273)$, 1,7-PHEN (20 $\mu \mathrm{M} ; n=279)$, or TIMP3 (400 nM; $n=302$ ). Final inhibitor concentrations were based on preliminary dose-response experiments. The CF and $\mathrm{Cl}$ oocytes were co-incubated with $1.25 \times 10^{5}$ spermatozoa $/ \mathrm{ml}$ for $6 \mathrm{~h}\left(39^{\circ} \mathrm{C}, 5 \% \mathrm{CO}_{2}\right)$. Before fixation, all groups were vortexed to remove excess spermatozoa and cultured during $18 \mathrm{~h}$. After Hoechst staining, the oocytes were evaluated for fertilization parameters as described earlier.

\section{Effect of 1,10-PHEN on sperm-zona binding}

In vitro matured COCs were denuded by vortexing with $0.1 \%$ $(\mathrm{w} / \mathrm{v})$ hyaluronidase in HEPES-TM and randomly assigned to two groups of 20 oocytes (three replicates). The first group was fertilized in standard fertilization medium (control), the second group in the presence of $20 \mu \mathrm{M} 1,10-\mathrm{PHEN}$. For unambiguous counting of the number of spermatozoa bound to the ZP, oocytes were co-incubated with $0.25 \times 10^{5}$ spermatozoa $/ \mathrm{ml}$ $\left(39{ }^{\circ} \mathrm{C}, 5 \% \mathrm{CO}_{2}\right)$. After 4 h of co-incubation (Fazeli et al. 1995), the oocytes were washed five times in PBS to remove loosely bound spermatozoa, and subsequently fixed and stained with Hoechst 33342. Of each oocyte, the average of two counts was taken into account as the number of sperm bound to the ZP.

\section{Evaluation of sperm membrane integrity, sperm motility, and acrosomal status}

To exclude a possible negative influence on spermatozoa, parameters related to sperm quality were evaluated over time in the presence of protease inhibitor concentrations that were used in the experiments. Sperm membrane integrity, motility, and the acrosomal status were evaluated at different time points during incubation. Boar semen samples were diluted in HEPESTM to obtain a concentration of $20 \times 10^{6}$ spermatozoa $/ \mathrm{ml}$ and warmed in an incubator at $37^{\circ} \mathrm{C}$. After $30 \mathrm{~min}$ of warming, aliquots of diluted semen were incubated with and without the protease inhibitor concerned. Then at different time points $(0,1,4$, and $6 \mathrm{~h})$, one aliquot per test group was evaluated for membrane integrity. Membrane integrity was assessed using eosin-nigrosin staining, which is considered a reliable and feasible technique (Maes et al. 2011). The average of three counts of 100 spermatozoa per aliquot was taken into account. Total and progressive motility were assessed by means of computer-assisted sperm analysis (Hamilton Thorne, Beverly, MA, USA). After $0,1,2$, and $6 \mathrm{~h}$ of incubation, $5 \mu \mathrm{l}$ of each sperm fraction was mounted on a Makler Counting Chamber and maintained at $37^{\circ} \mathrm{C}$ using a minitherm stage warmer. Five randomly selected microscopic fields were scanned for five times each. Statistical analysis was performed using the mean of the five scans of each microscopic field.

Acrosomal status was evaluated using FITC-conjugated Pisum sativum agglutinin (PSA-FITC; L0770, Sigma-Aldrich). Frozen epididymal semen was thawed for $60 \mathrm{~s}$ in a water bath at $38^{\circ} \mathrm{C}$ and spermatozoa were washed by centrifugation $(3 \mathrm{~min}$ at $390 \mathrm{~g}$ ) in $9.5 \mathrm{ml}$ Androhep. The sperm pellet was resuspended in FERT-TALP to obtain a sperm 
concentration of $2 \times 10^{6}$ spermatozoa $/ \mathrm{ml}$ and divided into six aliquots of $1 \mathrm{ml}$. Aliquot 1 without inhibitor was used as negative control. A second aliquot was supplemented with $1 \mu \mathrm{M}$ A23187 calcium ionophore as full response control. Sperm aliquots 3 and 4 were treated with $20 \mu \mathrm{M}$ 1,10-PHEN, aliquots 5 and 6 were treated with 400 nM TIMP3. Sperm aliquots 4 and 6 were then supplemented with $1 \mu \mathrm{M}$ A23187 calcium ionophore to evaluate the response in the presence of a protease inhibitor. All incubations were carried out for $1 \mathrm{~h}$ at $39^{\circ} \mathrm{C}$ in a $5 \% \mathrm{CO}_{2}$ incubator. Subsequently, $1 \mu \mathrm{l}$ Hoechst 33342 (Molecular Probes) was added to each aliquot and sperm aliquots were incubated for $3 \mathrm{~min}$ at $37^{\circ} \mathrm{C}$. After centrifugation at $720 \mathrm{~g}$ for $10 \mathrm{~min}$, the supernatant was removed and the sperm pellets were resuspended in $50 \mu \mathrm{l} 96 \%$ ethyl alcohol and incubated for $30 \mathrm{~min}$ at $4{ }^{\circ} \mathrm{C}$. Afterward, $15 \mu \mathrm{l}$ of each sperm aliquot was smeared on a glass slide and air-dried. Then $15 \mu \mathrm{l}$ PSA-FITC (2 mg PSA-FITC diluted in $2 \mathrm{ml} \mathrm{PBS)} \mathrm{was} \mathrm{added.} \mathrm{The} \mathrm{glass} \mathrm{slides}$ were kept for $15 \mathrm{~min}$ at $4{ }^{\circ} \mathrm{C}$, washed five times with deionized water, and air-dried. At least 100 spermatozoa in each sperm aliquot were evaluated using a Leica DMR fluorescence microscope (Leica Microsystems).

All spermatozoa were stained with Hoechst. The acrosomal region of acrosome-intact spermatozoa was PSA-FITC positive and labeled green, while acrosome-reacted spermatozoa retained only an equatorial labeled band with little or no labeling of the anterior head region.

\section{Visualization of spermatozoa within the cumulus oophorus}

Frozen-thawed spermatozoa were washed by centrifugation ( $3 \mathrm{~min}$ at $390 \mathrm{~g}$ ) in $9.5 \mathrm{ml}$ Androhep. The sperm pellet was diluted in HEPES-TM to a concentration of $20 \times 10^{6}$ spermatozoa/ml and spermatozoa were incubated with $250 \mathrm{nM}$ Mitotracker (M7511, Molecular Probes) during $20 \mathrm{~min}$ at $38{ }^{\circ} \mathrm{C}$. Subsequently, spermatozoa were washed twice by centrifugation in HEPES-TM (3 min at $390 \mathrm{~g}$ ) and diluted in FERT-TALP. The mitotracker-labeled spermatozoa were added to $25 \mu \mathrm{l}$ fertilization droplets containing in vitro matured COCs (1 COC per droplet) resulting in a final concentration of $1.25 \times$ $10^{5} \mathrm{spermatozoa} / \mathrm{ml}$. Gamete co-incubation was performed in standard FERT-TALP medium and in FERT-TALP with $20 \mu \mathrm{M}$ 1,10-PHEN. After 2, 4, and $6 \mathrm{~h}$ of incubation, COCs were washed individually in PBS and fixed with $2 \%$ paraformaldehyde for $20 \mathrm{~min}$ at room temperature. To stain the nuclei, COCs were incubated with $10 \mu \mathrm{g} / \mathrm{ml}$ Hoechst 33342 for $3 \mathrm{~min}$ at room temperature, followed by two washes in PBS. Each COC was mounted in glycerol with DABCO and evaluated for the presence of mitotracker-labeled spermatozoa in the cumulus oophorus using a Leica DMR fluorescence microscope (Leica Microsystems).

\section{Statistical analysis}

Differences in percentages of total fertilization and polyspermy rate were analyzed by means of binary logistic regression. ANOVA was used to evaluate the SPI and the differences in mean number of spermatozoa bound to the ZP. Differences in membrane integrity, sperm motility, and acrosomal status were evaluated using repeated measures ANOVA. Hypothesis testing was performed using a significance level of $5 \%$ (two-sided tests; SPSS 16.0).

\section{Declaration of interest}

The authors declare that there is no conflict of interest that could be perceived as prejudicing the impartiality of the research reported.

\section{Funding}

This study was supported by Research Foundation Flanders (grant numbers 1.1.450.09.N.00 and 1.1.450.11.N.01).

\section{Acknowledgements}

The authors wish to thank Isabel Lemahieu and Petra Van Damme for their excellent technical assistance. Frozen epididymal boar semen was kindly provided by Dr D Rath, Institute of Animal Science and Animal Behaviour, Mariensee, Germany.

\section{References}

Amour A, Slocombe PM, Webster A, Butler M, Knight CG, Smith BJ, Stephens PE, Shelley C, Hutton M, Knauper V et al. 1998 TNF- $\alpha$ converting enzyme (TACE) is inhibited by TIMP-3. FEBS Letters 435 39-44. (doi:10.1016/S0014-5793(98)01031-X)

Amour A, Knight CG, Webster A, Slocombe PM, Stephens PE, Knauper V, Docherty AJ \& Murphy G 2000 The in vitro activity of ADAM-10 is inhibited by TIMP-1 and TIMP-3. FEBS Letters 473 275-279. (doi:10.1016/S0014-5793(00)01528-3)

Amour A, Knight CG, English WR, Webster A, Slocombe PM, Knauper V, Docherty AJ, Becherer JD, Blobel CP \& Murphy G 2002 The enzymatic activity of ADAM 8 and ADAM9 is not regulated by TIMPs. FEBS Letters 524 154-158. (doi:10.1016/S0014-5793(02)03047-8)

Beynon RJ \& Bond JS 2001. In Proteolytic Enzymes: a Practical Approach, p 324-325. Oxford, New York: Oxford University Press.

Bijttebier J, Van Soom A, Meyer E, Mateusen B \& Maes D 2008 Preovulatory follicular fluid during in vitro maturation decreases polyspermic fertilization of cumulus-intact porcine oocytes in vitro maturation of porcine oocytes. Theriogenology 70 715-724. (doi:10.1016/j.theriogenology.2008.04.046)

Bond JS \& Butler PE 1987 Intracellular proteases. Annual Review of Biochemistry 56 333-364. (doi:10.1146/annurev.bi.56.070187.002001)

Brooks PC, Stromblad S, Sanders LC, von Schalscha TL, Aimes RT, StetlerStevenson WG, Quigley JP \& Cheresh DA 1996 Localization of matrix metalloproteinase MMP-2 to the surface of invasive cells by interaction with integrin $\alpha \vee \beta 3$. Cell 85 683-693. (doi:10.1016/S00928674(00)81235-0)

Brown CR \& Cheng WT 1985 Limited proteolysis of the porcine zona pellucida by homologous sperm acrosin. Journal of Reproduction and Fertility 74 257-260. (doi:10.1530/jrf.0.0740257)

Brown HM, Dunning KR, Robker RL, Boerboom D, Pritchard M, Lane M \& Russell DL 2010 ADAMTS1 cleavage of versican mediates essential structural remodeling of the ovarian follicle and cumulus-oocyte matrix during ovulation in mice. Biology of Reproduction 83 549-557. (doi:10.1095/biolreprod.110.084434)

Buchman-Shaked O, Kraiem Z, Gonen Y \& Goldman S 2002 Presence of matrix metalloproteinases and tissue inhibitor of matrix metalloproteinase in human sperm. Journal of Andrology 23 702-708. 
Correa LM, Cho C, Myles DG \& Primakoff P 2000 A role for a TIMP-3sensitive, $\mathrm{Zn}(2+)$-dependent metalloprotease in mammalian gamete membrane fusion. Developmental Biology 225 124-134. (doi:10.1006/ dbio.2000.9825)

D'Alessandris C, Canipari R, Di Giacomo M, Epifano O, Camaioni A, Siracusa G \& Salustri A 2001 Control of mouse cumulus cell-oocyte complex integrity before and after ovulation: plasminogen activator synthesis and matrix degradation. Endocrinology 142 3033-3040. (doi:10.1210/en.142.7.3033)

Evans JP 2001 Fertilin $\beta$ and other ADAMs as integrin ligands: insights into cell adhesion and fertilization. BioEssays 23 628-639. (doi:10.1002/bies. 1088)

Fabrega A, Guyonnet B, Dacheux JL, Gatti JL, Puigmule M, Bonet S \& Pinart E 2011 Expression, immunolocalization and processing of fertilins ADAM-1 and ADAM-2 in the boar (Sus domesticus) spermatozoa during epididymal maturation. Reproductive Biology and Endocrinology 996. (doi:10.1186/1477-7827-9-96)

Fazeli AR, Holt C, Steenweg W, Bevers MM, Holt WV \& Colenbrander B 1995 Development of a sperm hemizona binding assay for boar semen. Theriogenology 44 17-27. (doi:10.1016/0093691X(95)00144-W)

Honda A, Siruntawineti J \& Baba T 2002 Role of acrosomal matrix proteases in sperm-zona pellucida interactions. Human Reproduction Update 8 405-412. (doi:10.1093/humupd/8.5.405)

Jin M, Fujiwara E, Kakiuchi Y, Okabe M, Satouh Y, Baba SA, Chiba K \& Hirohashi N 2011 Most fertilizing mouse spermatozoa begin their acrosome reaction before contact with the zona pellucida during in vitro fertilization. PNAS 108 4892-4896. (doi:10.1073/pnas. 1018202108)

Kashiwagi M, Tortorella M, Nagase H \& Brew K 2001 TIMP-3 is a potent inhibitor of aggrecanase 1 (ADAM-TS4) and aggrecanase 2 (ADAM-TS5). Journal of Biological Chemistry 276 12501-12504. (doi:10.1074/jbc. C000848200)

Kim E, Yamashita M, Kimura M, Honda A, Kashiwabara S-I \& Baba T 2008 Sperm penetration through cumulus mass and zona pellucida. International Journal of Developmental Biology 52 677-682. (doi:10.1387/ijdb.072528ek)

Loechel F, Fox JW, Murphy G, Albrechtsen R \& Wewer UM 2000 ADAM 12-S cleaves IGFBP-3 and IGFBP-5 and is inhibited by TIMP-3. Biochemical and Biophysical Research Communications 278 511-515. (doi:10.1006/bbrc.2000.3835)

Maes D, Lopez-Rodriguez A, Rijsselaere T, Vyt P \& Soom AV 2011 Artificial insemination in pigs. In Artificial Insemination in Farm Animals, pp 87-94. Ed M Manafi. Rijeka, Croatia: InTech.

Maretzky T, Reiss K, Ludwig A, Buchholz J, Scholz F, Proksch E, de Strooper B, Hartmann D \& Saftig P 2005 ADAM10 mediates E-cadherin shedding and regulates epithelial cell-cell adhesion, migration, and $\beta$-catenin translocation. PNAS 102 9182-9187. (doi:10. 1073/pnas.0500918102)

Nagano O \& Saya H 2004 Mechanism and biological significance of CD44 cleavage. Cancer Science 95 930-935. (doi:10.1111/j.1349-7006.2004. tb03179.x)

Nagano O, Murakami D, Hartmann D, De Strooper B, Saftig P, Iwatsubo T, Nakajima M, Shinohara M \& Saya H 2004 Cell-matrix interaction via CD44 is independently regulated by different metalloproteinases activated in response to extracellular $\mathrm{Ca}(2+)$ influx and PKC activation. Journal of Cell Biology 165 893-902. (doi:10.1083/ jcb.200310024

Nagase H, Visse R \& Murphy G 2006 Structure and function of matrix metalloproteinases and TIMPs. Cardiovascular Research 69 562-573. (doi:10.1016/j.cardiores.2005.12.002)

Peschon JJ, Slack JL, Reddy P, Stocking KL, Sunnarborg SW, Lee DC, Russell WE, Castner BJ, Johnson RS, Fitzner JN et al. 1998 An essential role for ectodomain shedding in mammalian development. Science $\mathbf{2 8 2}$ 1281-1284. (doi:10.1126/science.282.5392.1281)

Petters RM \& Wells KD 1993 Culture of pig embryos. Journal of Reproduction and Fertility. Supplement 48 61-73.

Polakoski KL \& McRorie RA 1973 Boar acrosin. II. Classification, inhibition, and specificity studies of a proteinase from sperm acrosomes. Journal of Biological Chemistry 248 8183-8188.
Polakoski KL, McRorie RA \& Williams WL 1973 Boar acrosin. I. Purification and preliminary characterization of a proteinase from boar sperm acrosomes. Journal of Biological Chemistry 248 8178-8182.

Primakoff P \& Myles DG 2002 Penetration, adhesion, and fusion in mammalian sperm-egg interaction. Science 296 2183-2185. (doi:10.1126/science.1072029)

Primakoff P, Hyatt H \& Tredick-Kline J 1987 Identification and purification of a sperm surface protein with a potential role in sperm-egg membrane fusion. Journal of Cell Biology 104 141-149. (doi:10.1083/ jcb.104.1.141)

Rath D, Long CR, Dobrinsky JR, Welch GR, Schreier LL \& Johnson LA 1999 In vitro production of sexed embryos for gender preselection: high-speed sorting of X-chromosome-bearing sperm to produce pigs after embryo transfer. Journal of Animal Science 77 3346-3352.

Rawlings ND, Barrett AJ \& Bateman A 2012 MEROPS: the database of proteolytic enzymes, their substrates and inhibitors. Nucleic Acids Research 40 D343-D350. (doi:10.1093/nar/gkr987)

Reiss K \& Saftig P 2009 The "a disintegrin and metalloprotease" (ADAM) family of sheddases: physiological and cellular functions. Seminars in Cell \& Developmental Biology 20 126-137. (doi:10.1016/j.semcdb. 2008.11.002)

Rodriguez-Manzaneque JC, Westling J, Thai SN, Luque A, Knauper V, Murphy G, Sandy JD \& Iruela-Arispe ML 2002 ADAMTS1 cleaves aggrecan at multiple sites and is differentially inhibited by metalloproteinase inhibitors. Biochemical and Biophysical Research Communications 293 501-508. (doi:10.1016/S0006-291X (02)00254-1)

Russell DL, Doyle KM, Ochsner SA, Sandy JD \& Richards JS 2003 Processing and localization of ADAMTS-1 and proteolytic cleavage of versican during cumulus matrix expansion and ovulation. Journal of Biological Chemistry 278 42330-42339. (doi:10.1074/jbc.M300 519200)

Shimada M, Nishibori M, Yamashita Y, Ito J, Mori T \& Richards JS 2004 Down-regulated expression of $\mathrm{A}$ disintegrin and metalloproteinase with thrombospondin-like repeats- 1 by progesterone receptor antagonist is associated with impaired expansion of porcine cumulus-oocyte complexes. Endocrinology 145 4603-4614. (doi:10.1210/en.20040542)

Shiomi T, Lemaitre V, D'Armiento J \& Okada Y 2010 Matrix metalloproteinases, a disintegrin and metalloproteinases, and a disintegrin and metalloproteinases with thrombospondin motifs in non-neoplastic diseases. Pathology International 60 477-496. (doi:10.1111/j.14401827.2010.02547.x)

Stetler-Stevenson WG \& Yu AE 2001 Proteases in invasion: matrix metalloproteinases. Seminars in Cancer Biology 11 143-153. (doi:10.1006/scbi.2000.0365)

Sun TT, Chung CM \& Chan HC 2011 Acrosome reaction in the cumulus oophorus revisited: involvement of a novel sperm-released factor NYD-SP8. Protein \& Cell 2 92-98. (doi:10.1007/s13238-011-1022-5)

Sutovsky P, Manandhar G, McCauley TC, Caamano JN, Sutovsky M, Thompson WE \& Day BN 2004 Proteasomal interference prevents zona pellucida penetration and fertilization in mammals. Biology of Reproduction 71 1625-1637. (doi:10.1095/biolreprod.104.032532)

Van Soom A, Tanghe S, De Pauw I, Maes D \& de Kruif A 2002 Function of the cumulus oophorus before and during mammalian fertilization. Reproduction in Domestic Animals 37 144-151. (doi:10.1046/j.14390531.2002.00345.x)

Wang WM, Ge G, Lim NH, Nagase H \& Greenspan DS 2006 TIMP-3 inhibits the procollagen N-proteinase ADAMTS-2. Biochemical Journal 398 515-519. (doi:10.1042/BJ20060630)

Yamashita Y, Kawashima I, Yanai Y, Nishibori M, Richards JS \& Shimada M 2007 Hormone-induced expression of tumor necrosis factor $\alpha$-converting enzyme/a disintegrin and metalloprotease-17 impacts porcine cumulus cell oocyte complex expansion and meiotic maturation via ligand activation of the epidermal growth factor receptor. Endocrinology 148 6164-6175. (doi:10.1210/en.2007-0195)

Yi YJ, Manandhar G, Sutovsky M, Li R, Jonakova V, Oko R, Park CS, Prather RS \& Sutovsky P 2007 Ubiquitin C-terminal hydrolase-activity is involved in sperm acrosomal function and anti-polyspermy defense during porcine fertilization. Biology of Reproduction 77 780-793. (doi:10.1095/biolreprod.107.061275) 
Yin L, Chung CM, Huo R, Liu H, Zhou C, Xu W, Zhu H, Zhang J, Shi Q, Wong HYC et al. 2009 A sperm GPI-anchored protein elicits spermcumulus cross-talk leading to the acrosome reaction. Cellular and Molecular Life Sciences 66 900-908. (doi:10.1007/s00018-009-8482-2)

Yokoo M, Tienthai P, Kimura N, Niwa K, Sato E \& Rodriguez-Martinez H 2002 Localisation of the hyaluronan receptor CD44 in porcine cumulus cells during in vivo and in vitro maturation. Zygote 10 317-326. (doi:10.1017/ S0967199402004057)

Yokoo M, Shimizu T, Kimura N, Tunjung WAS, Matsumoto $H$, Abe $H$, Sasada H, Rodriguez-Martinez H \& Sato E 2007 Role of the hyaluronan receptor CD44 during porcine oocyte maturation. Journal of Reproduction and Development 53 263-270. (doi:10.1262/jrd.18047)
Yu Q \& Stamenkovic I 1999 Localization of matrix metalloproteinase 9 to the cell surface provides a mechanism for CD44-mediated tumor invasion. Genes and Development 13 35-48. (doi:10.1101/gad. 13.1.35)

Received 6 August 2012

First decision 6 September 2012

Accepted 16 October 2012 